\title{
Efectos de una competición de fútbol basada en la Liga Brave sobre el fair play, las orientaciones de meta, el esfuerzo y la presión-tensión en escolares de educación primaria
}

\section{Effects of a football competition based on the Brave League on fair play, goal orientation, effort and pressure-tension on primary school students}

\author{
Pablo Lis-Velado y Alejandro Carriedo
}

Departamento de Ciencias de la Educación. Facultad de Formación del Profesorado y Educación. Universidad de Oviedo (España)

\begin{abstract}
Resumen: Se examinó la repercusión de la novedosa Liga Brave (2015) sobre las orientaciones de meta, el fair play, el esfuerzo, y la presión-tensión en 92 estudiantes de 2 colegios (grupo control: $n=45$; grupo experimental: $n$ $=47)$ con una media de edad de 10,38 ańos $(D E=0,55)$. Durante 3 sesiones, el grupo control participó en una liga de fútbol sala tradicional, mientras que el grupo experimental participó en un sistema competitivo basado en el innovador formato de la Liga Brave (2015), donde las conductas de fair play determinan el orden en la clasificación. Se realizaron varias pruebas de contrastes de medias intra-sujetos, cuyos resultados reflejaron que el grupo experimental aumentó significativamente en la orientación a la tarea y en el esfuerzo percibido. Estos resultados sugieren que formatos competitivos como el que se propone podrían repercutir positivamente en la orientación a la tarea y en el esfuerzo durante las competiciones deportivas.

Palabras clave: Fútbol, Educación física, deporte educativo, clima motivacional.
\end{abstract}

Abstract: This research analyzed the Brave League (2015) effects on the goal orientation, the fair play, the effort and the pressure-tension on $92 \mathrm{pu}$ pils from two state schools (control group: $n=45$ and experimental group: $n=47)$ with an age average of 10.38 years $(S D=0.55)$. The Intervention program was applied to both groups in 3 sessions of one hour; the control group created an indoor football league, whereas the experimental group continued to elaborate a competitive system based on the Brave League (2015) format, where the conducts of fair play establish the order in the classification. Several contrast tests were taken of the subject average, which results showed that the trial group increased the orientation towards the task) and the perceived effort. These results infer that the competitive formats like the proposed could affect positively the orientation towards the task and the effort throughout the sports competitions.

Keywords: Football, Physical education, sport education, motivational climate.

\section{Introducción}

El deporte es un importante fenómeno social y cultural (véanse los elevados índices de audiencia televisiva que genera en nuestro país: Barlovento, 2017; Carriedo, González, \& López, 2019) que se ha convertido en uno de los contenidos más utilizados en las clases de educación física (Méndez, Fernández-Río, Méndez-Giménez, \& Prieto, 2015). Por esta y otras razones, el estudio y análisis del deporte en edad escolar ha creado tanto interés entre los investigadores (e.g., Cecchini, González, \& Montero, 2007, 2008; Gibbons, Ebbeck, \& Weiss, 1995; Schwamberger \& Curtner-Smith, 2016). El deporte tiene la capacidad de ser un medio educativo y transmisor de valores positivos, pero para que esto ocurra, es necesario que se desarrolle bajo las condiciones adecuadas (Cecchini et al., 2007, 2008; Cecchini, Fernández-Río, Méndez-Giménez, Cecchini, \& Martins, 2014; González, 2008; Orts, 2013; Giménez, Abad, \& Robles, 2009). Barbero (1989) fue uno de los primeros autores que destacó la importancia de un cambio en la didáctica de los deportes en

Dirección para correspondencia [Correspondence address]: Alejandro Carriedo. E-mail: carriedoalejandro@uniovi.es el ámbito educativo e introdujo el término de "depuración deportiva” con la intención de convertir esta enseñanza en un hecho educativo. Barbero (1989) propuso que el deporte: a) no limitase la participación por niveles de habilidad; b) que su finalidad no fuese exclusivamente la mejora de las habilidades motrices (desarrollo cognitivo, interacción social); c) que no incidiese solo en el resultado sino en las experiencias corporales; d) que buscase la participación y la creatividad; e) que se pudiesen cambiar o modificar las condiciones de juego; f) que presentase la competición como un medio, no como un fin; g) que fomentase la actitud crítica; h) y que se recuperasen los juegos y deportes tradicionales. Sin embargo, a pesar de estas recomendaciones, Cecchini (2015) afirmó que la práctica deportiva, tal y como se está implementando en la actualidad, no desarrolla valores, es más, añadió que bajo determinadas circunstancias los resultados podían ser justamente los contrarios. De la misma manera varios autores han argumentado que el impacto negativo o positivo de la práctica deportiva en jóvenes está multideterminado y que depende de la orientación que le den las organizaciones de las prácticas deportivas, entrenadores, árbitros, padres, ami- 
gos y público, sin olvidar los modelos que ofrece el deporte profesional o de élite (Cruz, Anglès, Barros, \& Álvarez, 2001; Weinberg \& Gould, 2010; Castillo, García-Merita, Mercé, \& Balaguer, 2011).

Distintos autores han señalado el papel que podrían tener los medios de comunicación en el comportamiento de los aficionados (Barrero, 2007; Carriedo, Cecchini, \& González, 2018, 2019; Gómez, 2007; Vilches; 2004), y por lo tanto, en los jóvenes deportistas, quienes tienden a imitar las conductas agresivas de los profesionales (CSD, 2011; Smith, 1988). Por este motivo es necesario contar con herramientas que puedan mitigar la transmisión de valores negativos en el ámbito educativo, así como con iniciativas que favorezcan la iniciación deportiva desde el fair play (Pinheiro, 2013). En este sentido, distintos trabajos han tratado de intervenir en estas cuestiones a través de la teoría de las metas de logro (Ames, 1992; Nicholls, 1984, 1989). Esta teoría postula que en los contextos de logro de objetivos, como es el caso del deporte competitivo, existen dos dimensiones independientes que explican la percepción de éxito y de fracaso: la orientación a la tarea, basada en la mejora personal auto-referenciada; y la orientación al ego, cuando se concibe el éxito en función del resultado y uno se compara con los demás (Nicholls, 1984, 1989). Se ha descubierto que la primera de ellas se relaciona positivamente con la diversión y los motivos sociales (Castillo, Balaguer, y Duda, 2007), así como con la deportividad (Lemyre, Roberts, \& Ommundsen, 2002), la motivación intrínseca y las opiniones y conductas de fair play (Cecchini, González, López, \& Brustad, 2005). De acuerdo con Cervelló, Escartí, y Balagué (1999), la orientación a la tarea se asocia con patrones motivacionales más adaptativos, mientras que la orientación al ego se ha relacionado con patrones menos adaptativos. Goldstein e Iso-Ahola (2006) señalan que entre los jóvenes deportistas suele predominar una orientación hacia el ego.

Dentro del estudio de las variables motivacionales en el contexto de la educación física también se han analizado los niveles de esfuerzo, de presión-tensión percibida durante la práctica deportiva o de la diversión. Por ejemplo, se ha encontrado que los climas orientados a la tarea se relacionan con mayores niveles de confianza y diversión durante la práctica mientras que los orientados al ego se asocian con la ansiedad y el aburrimiento (Braithwaite, Spray, \& Warburton, 2011), que la motivación intrínseca predice el esfuerzo (Cecchini, Carriedo, \& Méndez-Giménez, 2019), que la diversión también es un buen predictor del esfuerzo y del aburrimiento de los jóvenes, así como que la desmotivación se relaciona positivamente con el aburrimiento y la presión-tensión, y negativamente con el esfuerzo (Méndez-Giménez, Fernández-Río, \& Cecchini, 2016).

El fair play puede definirse como juego limpio, que de acuerdo con el Diccionario de las Ciencias del Deporte (Aquesolo, 1992) sería el respeto y reconocimiento por las re- glas del juego, relacionarse correctamente con el adversario, mantener una actitud digna ante la victoria y la derrota, y promover la igualdad de oportunidades para todos los participantes durante la práctica deportiva, así como rechazar la violencia a cualquier precio y comprometerse a dar el máximo. Otro término afín es deportividad, que según Lee (1996) se fundamenta en el principio de justicia para todos, donde no existe la intención (ni fortuita, ni deliberada) de conseguir una ventaja deshonesta sobre el adversario.

La investigación sobre el juego limpio durante la práctica deportiva también se ha fundamentado en las teorías de la motivación. La investigación ha demostrado en repetidas ocasiones que las percepciones de clima de maestría se asocian positivamente con la orientación a la tarea, la diversión, el esfuerzo, las opiniones de fair play y negativamente con la ansiedad (Cecchini et al., 2007; Cecchini, González, López, $\&$ Brustad, 2005). Por su parte, los climas de ejecución se relacionan positivamente con la orientación al ego, la ansiedad, las opiniones favorables hacia el juego duro, el comportamiento antisocial (Boixadós \& Cruz, 1999; Guivernau \& Duda, 2002; Kavussanu, 2006; Ommundsen, Roberts, Lemyre, \& Treasure, 2003; Stephens, 2000; Stornes \& Ommundsen, 2004), la búsqueda de victoria y las opiniones y conductas antideportivas (Cecchini et al., 2005). Cruz (1992) señala que las posibles causas del deterioro del fair play en edad infantil y juvenil son: a) el aumento de las recompensas económicas por el éxito en el deporte infantil y juvenil; b) los valores, actitudes y conductas de entrenadores orientadas al éxito en las competiciones; c) las actitudes y conductas de padres y espectadores durante los partidos; d) el sistema de sanciones de algunos reglamentos que benefician al infractor (e.g., en el futbol se considera una falta útil y necesaria aquella que se hace con el objetivo de cortar un contrataque peligroso). Con base en estas cuestiones, Cruz (1992) propone que las líneas de actuación deberían basarse en la necesidad de convertir el deporte en un hecho educativo mediante el desarrollo de la cooperación y fomentar entre los niños y niñas estrategias de razonamiento moral en las clases de educación física; en mejorar la formación y el asesoramiento de entrenadores de niños y niñas en edad escolar; en formar y asesorar a árbitros y organizadores de competiciones infantiles; y en la promoción de la educación deportiva de la población, mediante campañas nacionales e internacionales sobre el fair play. En este sentido, Sáenz, Gimeno, Gutiérrez, y Garay (2012) seńalan que sería muy interesante que los programas, además de proponer estrategias de prevención, planteasen al mismo tiempo acciones de intervención y rehabilitación, ya que la prevención es más efectiva cuando todavía no se han dado conductas antideportivas o violentas, pero la realidad es que los alumnos ya están condicionados negativamente.

Se ha sugerido que el deporte de élite crea, entre otros, valores negativos en la conducta de los niños (Pinheiro, Ca- 
meiro, \& Sequeira, 2014). Por ello, se han propuesto diferentes intervenciones para mejorar las conductas antideportivas y fomentar el fair play en edad escolar (e.g., Brunelle et al., 2005; Cecchini et al., 2003; Cecchini, et al., 2008). Los estudios que han analizado las posibilidades de las intervenciones basadas en este tipo de propuestas han obtenido resultados prometedores (e.g., Cecchini et al., 2008; Danish, 1997; Danish \& Nellen, 1997; Ennis, 1999; Miller, Bredemeier, \& Shields, 1997; Lamoneda, Córdoba, Huertas, \& García, 2015). Sin embargo, se observa la necesidad de seguir investigando sobre este tema.

Recientemente, la Liga Brave (2015) ha hecho un esfuerzo por tratar de reducir el comportamiento antideportivo y agresivo entre los jugadores de fútbol más jóvenes. Proponen un formato de competición que tiene su origen en la popularmente conocida Liga de Juegos Escolares de la Vega Baja impulsada en sus inicios por el Ayuntamiento de Catral (Alicante) y respaldada desde 2011 por el director del futbol base del Elche CF. Se le conoce actualmente como Liga Brave y su principal objetivo es promover y premiar el juego limpio entre los equipos participantes, permitiendo crear un modelo de trasmisión de valores positivos y adecuados para la convivencia y el respeto a través del fútbol por medio de un sistema de puntuación que evalúa bajo diferentes criterios, el juego limpio y las conductas antideportivas encontradas en los equipos en el trascurso de los diferentes partidos del campeonato. Para esto han creado un sistema de dos clasificaciones independientes, una donde se reflejan los puntos conseguidos por las victorias (3 puntos), derrotas (0 puntos) o empates (1 punto), y otra donde se plasman las conductas contrarias al fair play. A esta última clasificación se le concede mucho más valor que a la primera cuando finaliza el campeonato.

Por lo tanto, con base en estos antecedentes, el objetivo principal de este estudio fue el de analizar los efectos de una competición de fútbol basada en la Liga Brave (2015) en las actitudes de fair play, en las orientaciones de meta, en el esfuerzo, y en la presión-tensión en estudiantes de $5^{\circ}$ curso de educación primaria.

\section{Método}

\section{Participantes}

Esta investigación parte de un diseño cuasi-experimental pre-test post-test con grupo control en la que participaron 92 estudiantes de $5^{\circ}$ curso de educación primaria que pertenecían a dos colegios de titularidad pública de una ciudad del norte de Espańa (50 hombres y 42 mujeres). La edad de los participantes oscilaba entre 10 y 12 años $(M=10,38, D E$ $=0,55)$. Mediante asignación aleatoria, los estudiantes de un centro educativo fueron asignados al grupo control $(n=45$;
28 hombres y 17 mujeres) y los estudiantes del otro centro fueron asignados al grupo experimental $(n=47 ; 22$ hombres y 25 mujeres).

\section{Instrumentos y variables de medida}

Fair play. La Escala de Actitudes de Fair play (Cruz et al., 1996) es un cuestionario de 23 ítems divididos en tres subescalas: 12 de juego duro (e.g., "Es aceptable que un jugador reaccione violentamente cuando ha sido objeto de falta"), 6 de importancia de la victoria (e.g., "en el fútbol el resultado final es lo más importante), y 5 de divertimento (e.g., "en el fútbol divertirse jugando es lo más importante). Los participantes respondieron al encabezado: "pienso que...”. Este instrumento, que ha sido utilizado en investigaciones realizadas con escolares, ha mostrado adecuados índices de fiabilidad para las subescalas de juego duro: $\alpha=, 84$, victoria: $\alpha=, 73$, y divertimento: $\alpha=, 70$ (Ceccchini, Montero, \& Peña, 2003).

Orientaciones de meta. Se utilizó la versión en español (Cervelló et al., 1999) del Cuestionario de Percepción de Éxito (POSQ: Roberts \& Balagué, 1991; Roberts, Treasure \& Balagué, 1998) para medir las orientaciones de meta de los jóvenes discentes en las clases de Educación Física. El inventario se encuentra compuesto por 12 ítems, 6 de los cuales responden al factor orientación hacia la tarea (e.g., "trabajo duro") y los otros 6, al factor orientación hacia el ego del alumno (e.g., "soy el mejor"). Las respuestas respondían a la raíz "Cuando juego al fútbol en Educación Física siento que tengo éxito cuando...". La fiabilidad de dicho cuestionario ha sido demostrada en diferentes estudios llevados a cabo tanto en el ámbito deportivo (e.g., Treasure \& Roberts, 1994) como en el contexto de educación física en la etapa de primaria, obteniendo un alfa de ,70 para la subescala tarea y de ,80 para la subescala ego (Carriedo, 2015).

Presión-tensión y esfuerzo. Para medir la presión-tensión y el esfuerzo percibido por los estudiantes se utilizaron dos de las 4 subescalas del Cuestionario de motivación intrínseca (Intrinsic Motivation Inventory-IMI) de McAuley, Duncan, y Tammen (1989), que fue traducido y validado al castellano por Escartí y Gutiérrez (2001). Las subescalas utilizadas fueron: esfuerzo-importancia (4 ítems: e.g., "me esfuerzo mucho”) y tensión-presión (4 ítems: e.g., me siento muy tenso cuando participo"). Los estudiantes respondieron a la raíz “Cuando juego al fútbol en educación física...". La fiabilidad de cada subescala en el trabajo de McAuley et al. (1989) fue de ,84, y ,68 para el esfuerzo y presión/tensión, respectivamente.

Las preguntas de los tres instrumentos fueron respondidas mediante una escala Likert que oscilaba entre 1 (totalmente en desacuerdo) y 5 (totalmente de acuerdo). 


\section{Procedimiento}

En primer lugar se obtuvo el consentimiento del comité de ética de la Universidad. Después, se obtuvo el permiso de la dirección de los centros seleccionados, y finalmente se recogió el consentimiento informado de los familiares de los participantes.

Mediante asignación aleatoria se decidió que los estudiantes de $5^{\circ}$ curso de un colegio participasen en una liga de fútbol mediante un sistema competitivo tradicional (i.e., grupo control) y que los estudiantes del otro centro hicieran lo propio mediante el sistema propuesto por la Liga Brave (2015; i.e., grupo experimental). Para llevar a cabo esta investigación se necesitaron 3 sesiones de educación física por cada uno de los grupos de $5^{\circ}$ de Primaria que participaron en el estudio. Los alumnos rellenaron los cuestionarios de manera anónima y voluntaria antes y después de realizar la intervención. En ambos casos recibieron instrucciones sobre la importancia de ser sinceros y se les aseguró que sus respuestas serían completamente confidenciales. Asimismo, se les instó a pedir ayuda en caso necesario. En función del número de alumnos de cada grupo-clase, con la ayuda del maestro de educación física se organizaron entre 3 y 4 equipos (teniendo en cuenta las habilidades motrices de los alumnos para que estos fuesen equilibrados) para participar en una liga de fútbol sala que se desarrolló durante un periodo de 6 jornadas de competición con partidos de 12 minutos de duración. En el grupo control participaron en un campeonato de liga convencional donde la clasificación obedecía a los resultados que determinaban los goles conseguidos en cada partido. Por cada partido ganado obtenían 3 puntos, por los empatados conseguían 1 punto, y cuando perdían no obtenían ningún punto. También se registraban los goles a favor y los goles en contra. En el grupo experimental se procedió de la misma manera, con la peculiaridad de que participaron en un campeonato basado en el formato de la Liga Brave (2015). En este formato competitivo se caracteriza porque existen dos tipos de clasificaciones, una que valora el Fair Play de los alumnos y otra idéntica a la utilizada en el grupo control (i.e., victoria $=3$ puntos, empate $=1$ punto, derrota $=0$ puntos). En la primera clasificación se registraron las acciones anti-deportivas que cometía cada equipo (e.g., protestar una decisión del árbitro, juego peligroso, discutir con algún compañero, agresiones...), quedando en primer lugar aquel equipo que cometiese menos conductas contrarias al fair play. En esta formato se declara vencedor al equipo que queda primero en la clasificación fair play, y solo en caso de empate se recurriría a la clasificación convencional. Estas conductas antideportivas son señaladas por el árbitro mediante una tarjeta marrón, la cual se puede sacar también fuera del partido para evitar que los alumnos protesten jugadas acontecidas en el encuentro disputado. Antes de cada sesión se recordaba la importancia de adoptar actitudes deportivas a lo largo de los partidos. El investigador formó al maestro de educación física para que ambos pudieran arbitrar dos partidos simultáneos.

\section{Análisis de datos}

Los datos fueron analizados mediante el programa estadístico IBM SPSS 22 para Windows. Se ejecutaron análisis descriptivos, análisis de consistencia interna según las recomendaciones de Cronbach (1951), y debido a que cada grupo tenía menos de 50 participantes, se utilizó el test de Shapiro Wilk para analizar la distribución de la muestra (Elliott \& Woodward, 2007). En función de la distribución observada se realizaron pruebas de contrastes de medias paramétricas o no paramétricas (i.e., prueba ty suma de rangos de Wilcoxon) con su correspondiente tamaño del efecto (i.e., $d$ de Cohen y $r$ de Rosenthal). Finalmente se calculó la diferencia porcentual por diferencia de medias (Hopkins, 2006) en las variables que ofrecieron resultados significativos. Antes de ejecutar estas pruebas se realizó un análisis de valores perdidos, el cual determinó que cada variable tenía una tasa inferior al 5\% de valores perdidos completamente al azar (MCAR), por lo que solo unos pocos valores perdidos fueron imputados mediante el algoritmo EM (Expectation-Maximization; Dempster, Laird, \& Rubin, 1977).

\section{Resultados}

\section{Análisis preliminares}

Todos los estudiantes participaron en al menos 2 de las 3 clases que duró la intervención. La prueba de Shapiro-Wilk indicó que los datos solo se distribuían con normalidad en la subescala victoria del grupo control (pre: $W(45)=, 972, p>$ ,05; post: $W(45)=, 966, p>, 05)$. Para evaluar la consistencia interna de las subescalas se utilizó el alfa de Cronbach, cuyos resultados fueron satisfactorios en todas las variables (Tabla 1). Finalmente se realizó la prueba $U$ de Mann-Whitney para comprobar las diferencias entre los dos grupos antes de la intervención, mostrando que el grupo control ( $m d=55,58$ ) tenía mayor aceptación por el juego duro que el grupo experimental $(m d=37,81), U=700, Z=-2,795, p=, 005, r=, 29$; y que el grupo experimental percibía mayor esfuerzo $(m d=$ $54,65)$ cuando practicaba fútbol en educación física que el grupo control ( $m d=37,99), U=767, Z=-2,295, p=, 022, r$ $=, 24$. El análisis de la importancia de la victoria mediante la prueba $t$ para muestra independientes mostró que el grupo control $(M=2,59, D E=0,88)$ le confería mayor importancia que el grupo experimental $(M=1,96, D E=0,60), t(90)=$ $4,016, p<, 001, d=.84$

Efectos de una competición de fútbol basada en la Liga Brave sobre el fair play, las.. SPORT TK, 8(2), 65-72 


\section{Diferencias pre-post intervención}

Para conocer el alcance de los cambios producidos por el protocolo de la Liga Brave (2015) en estudiantes de $5^{\circ}$ curso de educación primaria se realizaron varias pruebas intra-sujetos en cada una de las dos condiciones del estudio (grupo control y grupo de intervención). Las medias y las desviaciones estándar de los cambios producidos en todas las variables pueden verse en la Tabla 1. Los análisis de medidas repetidas mediante la suma de rangos de Wilcoxon mostraron mejoras significativas tras aplicar la intervención en la orientación a la tarea, $Z=-2,006, p=, 045, r=, 21$; $y$ en el esfuerzo, $Z=-2,906, p=$ ,004, $r=, 30$. No se encontraron diferencias significativas en las variables victoria, $Z=-0,559, p>, 05, r=, 06$; juego duro; $Z=-0,324, p>, 05, r=, 03$; diversión; $Z=-0,772, p>, 05, r=$ ,08; presión-tensión, $Z=-1,440, p>, 05, r=, 15$; y orientación al ego, $Z=-0,559, p>, 05, r=, 06$.

Los análisis de medidas repetidas realizados con la prueba $t$ mostraron que los estudiantes del grupo control reportaron resultados muy similares antes y después de la intervención en la variable victoria, $t(44)=, 521, p>, 05, d=0,04$. Del mismo modo, los análisis de medidas repetidas mediante la suma de rangos de Wilcoxon mostraron que en el grupo control no se apreciaron cambios significativos antes y después de la competición en el resto de las variables analizadas, juego duro, $Z=-0,582, p>, 05, r=, 06$; diversión, $(Z=-2,188, p$ $>, 05, r=, 23$; esfuerzo, $Z=-1,052, p>, 05, r=, 11$; presióntensión, $Z=-0,236, p>, 05, r=, 02$; orientación al ego, $Z=$ $-1,550, p>, 05, r=, 16$; orientación a la tarea, $Z=-0,103, p>$ $, 05, r=, 01$.

Los análisis inter-grupo posteriores a la intervención mostraron los mismos resultados que en los análisis preliminares, manteniéndose las diferencias significativas en el juego duro, $U=649, Z=-3,198, p<, 001, r=, 33$; y en el esfuerzo, $U=$ 674, $Z=-3,055, p=, 002, r=, 31$; y en la importancia en la victoria, $t(90)=2,935, p=, 004, d=.62$.

Tabla 1. Medias, desviaciones estándar y alfas de Cronbach de todas las variables analizadas en las dos condiciones.

\begin{tabular}{|c|c|c|c|c|c|c|c|}
\hline & \multicolumn{2}{|c|}{ Consistencia } & \multicolumn{2}{|c|}{ Liga Tradicional } & \multicolumn{2}{|c|}{ Liga Brave } & \multirow{3}{*}{$\begin{array}{l}\text { Diferencia } \\
\text { porcentual }\end{array}$} \\
\hline & Pre & Post & Pre & Post & Pre & Post & \\
\hline & $\alpha$ & $\alpha$ & $M(D E)$ & $M(D E)$ & $M(D E)$ & $M(D E)$ & \\
\hline Victoria & ,73 & ,79 & $2,59(0,88)$ & $2,55(0,96)$ & $1,96(0,60)$ & $2,02(0,73)$ & \\
\hline Juego duro &, 77 &, 78 & $2,11(0,64)$ & $2,14(0,64)$ & $1,78(0,55)$ & $1,75(0,57)$ & \\
\hline Diversión &, 74 &, 85 & $4,27(0,78)$ & $4,04(0,94)$ & $4,38(0,70)$ & $4,29(0,90)$ & \\
\hline O. Tarea &, 73 &, 81 & $3,99(0,75)$ & $4,01(0,77)$ & $4,15(0,65)$ & $4,24^{*}(0,83)$ & $2,12 \%$ \\
\hline O. Ego & ,87 &, 88 & $2,30(1,02)$ & $2,44(1,05)$ & $2,02(0,89)$ & $2,09(1,03)$ & \\
\hline Esfuerzo & ,70 & ,82 & $3,99(0,86)$ & $4,08(0,97)$ & $4,35(0,77)$ & $4,60 *(0,60)$ & $5,43 \%$ \\
\hline Presión-tensión &, 71 &, 72 & $2,42(1,07)$ & $2,46(1,08)$ & $2,29(1,06)$ & $2,08(0,89)$ & \\
\hline
\end{tabular}

Nota. ${ }^{* *} p<.001 .{ }^{*} p<.05$

\section{Discusión}

El objetivo principal de este estudio fue analizar los efectos de una competición de fútbol basada en la Liga Brave (2015) sobre las actitudes de fair play, las orientaciones de meta, el esfuerzo, y la presión-tensión en estudiantes de $5^{\circ}$ curso de educación primaria. El juego deportivo es un medio idóneo para observar y medir el desarrollo de valores personales y sociales ya que ofrece muchas posibilidades de interacción, especialmente en edades comprendidas entre los 10 y los 13 años por corresponderse con una etapa evolutiva donde hay mayor interés por la práctica deportiva y por las relaciones sociales (Brustad \& Arruza, 2002). Los resultados de este estudio sugieren que el formato Liga Brave (2015) puede generar cambios, en un tiempo relativamente corto, en la orientación hacia la tarea de los participantes y en el esfuerzo percibido. En el resto de variables no se encontraron diferencias significativas.
Varias investigaciones que han examinado los efectos de distintos tipos de intervenciones para incidir en el fair play y en la educación en valores en preadolescentes han observado que los participantes del grupo experimental tenían más diversión, menor importancia en la victoria y menor juego duro después de la intervención (Cecchini et al., 2008; Cecchini, Montero, \& Peńa, 2003). Los análisis de nuestros resultados señalan que el grupo experimental no puntuó diferente en ninguna de las variables asociadas al fair play. Lo que es consistente con otro estudio que no encontró diferencias significativas entre el principio y el final de una temporada en la que se analizaron los efectos de una intervención para promocionar el fair play en jóvenes futbolistas (Sampol, Rotger, Verdaguer, Conti, \& Álvarez, 2007). En nuestro caso, la ausencia de diferencias significativas podría explicarse de diferentes formas. Por ejemplo, podría deberse a la escasa duración de la intervención lo que podría haber supuesto una amenaza a la 
validez interna en la administración de la prueba originando que los resultados del post-test hayan estado influenciados por el pre-test. Otra explicación es que efectivamente, el formato Liga Brave (2015) no tenga un efecto en estas variables porque no deja de ser un formato competitivo, y en la competición un equipo gana y otros pierden. Esta podría ser una de las diferencias existentes entre los trabajos previos que sí han encontrado mejoras en el fair play en contextos educativos (Cecchini et al., 2003; Cecchini, Fernández, González, \& Arruza, 2008; Vidoni \& Ward, 2009) y una similitud con los que no han encontrado diferencias en contextos competitivos (e.g., Sampol, Rotger, Verdaguer, Conti, \& Álvarez, 2007).

Con respecto a las orientaciones de meta, solo se encontraron mejoras significativas en la orientación a la tarea entre el alumnado del grupo experimental. Así, la orientación al ego permaneció estable en ambos grupos. Este resultado es relevante porque varios autores han recomendado establecer una fuente motivacional centrada en la tarea para mejorar las actitudes y los comportamientos relacionados con el fair play (e.g., Boixados, Cruz, Torregrosa, \& Valiente, 2004; Ommundsen et al., 2003). Asimismo, este hallazgo es consistente con otras investigaciones que demostraron que el clima de maestría (valoración y enfatización por parte del profesor del esfuerzo, el aprendizaje, el dominio de la tarea y la participación de los individuos) se asociaba positivamente con la orientación a la tarea, la diversión, el esfuerzo, la competencia percibida, las opiniones de divertimento y los comportamientos positivos de fair play, y negativamente con la ansiedad (Cecchini et al., 2005). Es decir, el modo de actuar y el rol que desempeńa el árbitro en la Liga Brave (2015), así como el reglamento de dicha competición que fomenta las actitudes deportivas, podría favorecer el establecimiento de un clima de maestría ya que el árbitro/profesor debe valorar la participación, el aprendizaje, el esfuerzo y el dominio de la tarea. Con respecto al resto de variables, los análisis indicaron que no había diferencias significativas en ninguno de los grupos después de la intervención.

Diversos trabajos han demostrado que cuanto más tiempo se dedica a la práctica de un deporte de contacto medio como el fútbol, los deportistas muestran peores niveles de fair play y agresividad (Cecchini et al., 2007, 2008; Conroy, Silva, Newcomer, Walker, \& Johnson, 2001). Por lo tanto, considerando la temprana edad con la que niños y niñas se inician en la práctica de este tipo de deportes, la modificación de estas conductas puede suponer un desafío. Sin embargo, este estudio alienta al optimismo por varios motivos. Por un lado, se ha observado que aquellos chicos y chicas con una alta orientación a la tarea perciben que la diversión y los motivos sociales (e.g., ver a los amigos o hacer nuevos amigos) son muy importantes en su experiencia deportiva (Castillo et al., 2007). Asimismo, se ha comprobado que la orientación a la tarea se interrelaciona con los motivos intrínsecos y las dimensiones sociales de la experiencia deportiva (Castillo et al., 2007; Duda \& Nicholls, 1992; White \& Duda, 1994). Por otro lado, la orientación a la tarea ha sido relacionada con actitudes deportivas positivas, concretamente con la deportividad (Cecchini et al., 2007; Lemyre et al., 2002), el respeto por los convencionalismos sociales y la entrega personal en el deporte (Dunn \& Dunn, 1999), así como con la diversión durante la práctica deportiva (Braithwaite et al., 2011).

\section{Conclusiones}

Puede concluirse que los resultados de este primer estudio alientan al optimismo y sugieren que este formato competitivo es capaz de producir cambios en un periodo relativamente corto de tiempo en la orientación a la tarea y en el esfuerzo de los participantes sin alterar la diversión percibida durante la competición. Los múltiples beneficios que subyacen en la orientación de la tarea de los participantes de actividades deportivas permiten sugerir que las aplicaciones prácticas de este descubrimiento orientan sobre las posibilidades de sistemas competitivos que resalten la importancia del juego limpio y de la deportividad frente a la victoria, por lo que los docentes de educación física y los gestores deportivos deberían tratar de desarrollar e implementar formatos competitivos basados en las características de la Ligrabrave (20015). Sin embargo, a pesar de los resultados obtenidos, el presente estudio presenta una serie de limitaciones que deberían tenerse en cuenta en trabajos posteriores. La primera de ellas es el número de participantes. La segunda, el poco tiempo empleado en la intervención en cada uno de los grupos. Del mismo modo, la proximidad en el tiempo entre la medición del pre y del post podría haber distorsionado las respuestas de los sujetos de la segunda medida. Es posible que una intervención más prolongada en el tiempo hubiera proporcionado otro tipo de resultados. Asimismo, la actuación de árbitros que pueden tener diferentes criterios a la hora de interpretar el reglamento de la Liga Brave (2015) podría haber sido una variable extraña que debería controlarse, por lo que deberían plantearse investigaciones longitudinales que analizasen estas variables en el tiempo y con un solo árbitro o varios árbitros que actuasen mediante un contravalanceo. No obstante, debe resaltarse que, a diferencia del grupo experimental, en el grupo control no hubo diferencias significativas en ninguna variable. Con el mismo fin también se podría aplicar este programa en niños y adolescentes con diferentes rangos de edad, en diferentes contextos o deportes, o controlando otras variables extrańas como la práctica deportiva extraescolar. Finalmente, para confirmar la funcionalidad de este sistema de competición se podría utilizar instrumentos de observación para evaluar las distintas reacciones y comportamientos de los sujetos a lo largo de todo el campeonato, de esta manera 
se recopilaría más información que ayudaría a interpretar los efectos obtenidos y a seguir investigando a cerca de la impor- tancia de instaurar un sistema educacional que favoreciese las actitudes positivas de nuestros alumnos.

\section{Referencias}

1. Ames, C. (1992). Achievement goals, motivational climate, and motivational processes. En G. Roberts (Ed.), Motivation in sport and exercise (pp. 161-176). Champaing, IL: Human Kinetics.

2. Aquesolo, J. (1992). Diccionario de las Ciencias del Deporte. Málaga: Unisport.

3. Barbero, J.I. (1989). La educación Física, materia escolar socialmente construida. Perspectivas de la Actividad Física y el deporte, 2, 30-34.

4. Barlovento comunicación (2017). Análisis televisivo 2017. Recuperado de https:/www.barloventocomunicacion.es/images/publicaciones/ ANUALES/analisis-televisivo-2017-Barlovento.pdf

5. Barrero, J. (2007). El tratamiento de la violencia en el fútbol por la prensa deportiva. Doxa comunicación, 5, 141-157.

6. Boixadós, M., Cruz, J., Torregrosa, M., \& Valiente, L. (2004). Relationship among motivational climate, satisfaction, perceived ability and fair play attitudes in young soccer players. Journal of Applied Sport Psychology, 16, 301-317.

7. Boixadós, M., \& Cruz, J. (1999). Relaciones entre clima motivacional y satisfacción, percepción de habilidad y actitudes de fairplay en futbolistas jóvenes. Revista de Psicología Social Aplicada, 9, 45-64.

8. Braithwaite, R., Spray, C.M., \& Warburton, V.E. (2011). Motivational climate interventions in physical education: A meta-analysis. Psychology of Sport and Exercise, 12, 628-638.

9. Brunelle, J., Goulet, C., \& Arguin, H. (2005). Promoting respect for rules and injury prevention in ice hockey: Evaluation of the fair-play program. Medical Sport Science Journal, 8(3), 294-304.

10. Brustad, R., \& Arruza, J.A. (2002). Práctica deportiva y desarrollo social en jóvenes deportistas. En J.A. Arruza (Ed.), Nuevas perspectivas acerca del deporte educativo (pp. 25-41). Bilbao: Universidad del País Vasco.

11. Carriedo, A. (2015). Metas de logro, diversión y persistencia-esfuerzo en estudiantes de educación física durante una unidad didáctica sobre judo. Magister, 27(2), 51-58. doi:10.1016/j.magis.2015.12.001

12. Carriedo, A., Cecchini, J.A., \& González, C. (2018). Evaluación de la metapercepción de meta y funcionamiento moral en espectadores de fútbol. Revista Internacional de Medicina y Ciencias de la Actividad Física y el Deporte, 18(70), 341-359. doi:10.15366/rimcafd2018.70.009

13. Carriedo, A., Cecchini, J.A., \& González, C. (2019). Soccer in the mass media: Examining the role of metaperceptions of goal orientation on spectators' moral functioning. Journal of Human Sport and Exercise, 14(3), in press. doi:10.14198/jhse.2019.143.08

14. Carriedo, A., González, C., \& López, I. (2019). Hábitos de consumo y práctica deportiva en estudiantes universitarios. Journal of Sport and Health Research. In press.

15. Castillo, I., Balaguer, I., \& Duda, J. L. (2007). Las orientaciones de meta y los motivos de práctica deportiva en los jóvenes deportistas valencianos escolarizados. Revista de Psicología del Deporte, 9(12), 37-50.

16. Castillo, I., Balaguer, I., \& Duda, J.L. (2001). Perspectivas de meta de los adolescentes en el contexto académico. Psicothema, 13(1), 79-86.

17. Castillo, I., García-Merita, M., Mercé, J., \& Balaguer, I. (2011). El papel del entrenador en el desarrollo positivo de los deportistas en edad escolar. Actas del II Congreso del deporte en Edad Escolar (pp.149-153). Valencia: Fundación Deportiva Municipal. Recuperado el 17 de febrero de 2018 de http://www.deporteescolarvalencia.com/uploads/editor/ libro_actas_ii_congreso_deporte_escolar.pdf

18. Cecchini, J.A. (2015). El deporte y la educación en valores. Lección inaugural del curso 2015-16. Universidad de Oviedo.

19. Cecchini, J.A., Carriedo, A., \& Méndez-Giménez, A. (2019). Testing a circular, feedback model in physical education from self-determination theory. The Journal of Educational Research, 112(4), 473-482. doi:10.10 80/00220671.2018.1555788

20. Cecchini, J.A., Fernández, J., González, C., \& Arruza, J.A. (2008). Repercusiones del Programa Delfos de educación en valores a través del deporte en jóvenes escolares. Revista de educación, 346, 167-186.

21. Cecchini, J.A., González, C., López, J., \& Brustad, R.J. (2005). Relación del clima motivacional percibido con la orientación de meta, la motivación intrínseca y las opiniones y conductas de fair play. Revista Mexicana de Psicología, 22(2), 469-479.

22. Cecchini, J.A., González, C., \& Montero, J. (2007). Participación en el deporte y fair play. Psicothema, 19(1), 57-64.

23. Cecchini, J.A., González, C., \& Montero, J. (2008). Participación en el deporte, orientación de metas y funcionamiento moral. Revista Latinoamericana de Piscología, 40(3), 497-509.

24. Cecchini, J., Montero, J., \& Peña, V. (2003). Repercusiones del programa de intervención para desarrollar la responsabilidad personal y social sobre los comportamientos de fair-play y el autocontrol. Psicothema, 15, 631-637.

25. Cecchini, J.A., Méndez-Giménez, A., \& Fernández-Río, J. (2014). Meta-percepciones de competencia de terceros significativos, competencia percibida, motivación situacional y orientaciones de deportividad en jóvenes deportistas. Revista de Psicología del Deporte, 23(2), 285-293.

26. Cervelló, E.M., Escartí, A., \& Balagué, G. (1999). Relaciones entre la orientación de metas disposicional y la satisfacción con los resultados deportivos, las creencias sobre las cusas de éxito en deporte y la diversión con la práctica deportiva. Revista de Psicología del Deporte, 8, 7-19.

27. Conroy, D.E., Silva, J.M., Newcomer, R.R., Walker, B. W., \& Johnson, M.S. (2001). Personal and participatory socializers of the perceived legitimacy of aggressive behavior in sport. Aggressive behavior, 27(6), 405-418.

28. Cronbach, L.J. (1951). Coefficient alpha and the internal structure of tests. psychometrika, 16(3), 297-334.

29. Cruz, J. (1992). Causes de la violència en l'esport infantil i intervencions psicològiques per a promoure el fairplay. En Actes de les VII Jornades de l'Associació Catalana de Psicologia de l'Esport (pp.159-166). Barcelona: ACPE.

30. Cruz, J., Boixadòs, M., Valiente, L., \& Torregrosa, M. (2001). ¿¿Se pierde el "fairplay" y la deportividad en el deporte en edad escolar? Apunts. Educación física y deportes, 2(64), 6-16.

31. Cruz, J., Capdevila, L., Boixados, M., Pintanel, M., Alonso, C., Mimbrero, J., \& Torregosa, M. (1996). Identificación de conductas, actitudes y valores relacionados con el fairplay en deportistas jóvenes. Investigaciones en Ciencias del Deporte, 9, 37-87.

32. CSD. (2011). Estudio los hábitos deportivos de la población escolar en España. Consejo Superior de Deportes. Presidencia de Gobierno.

33. Danish, S.J. (1997). Going for the goal: A life skills program for adolescents. En G. Albee, y T. Gullotta (Eds.), Primary preventions works (pp. 291-312). Thousand Oaks, CA: Sage.

34. Danish, S.J., \& Nellen, V.C. (1997). New roles for sport psychologists: Teaching life skills through sport to at risk youth. Quest, 49,100-113.

35. Dempster, A.P., Laird, N.M., \& Rubin, D.B. (1977). Maximum likelihood estimation from incomplete data via the EM algorithm (with discussion). Journal of the Royal Statistical Society. Series B, 39(1), 1-38.

36. Duda, J. L. \& Nicholls, J.G. (1992). Dimensions of achievement motivation in schoolwork and sport. Journal of Educational Psychology, 84, $1-10$ 
37. Dunn, J.G., \& Dunn, J. (1999). Goal orientations, perceptions of aggression and sportspersonship in elite male youth ice hockey players. The Sport Psychologist, 13, 183-200.

38. Elliott, A.C., \& Woodward, W.A. (2007). Statistical analysis quick reference guidebook with SPSS examples. London: Sage Publications.

39. Ennis, C.D. (1999). Creating a culturally relevant curriculum for disengaged girls. Sport, Education and Society, 4, 31-49.

40. Escartí, A., \& Gutiérrez, M. (2001). Influence of the motivational climate in physical education on the intention to practice physical activity or sport. European Journal of Sport Sciences, 1(4), 1-12.

41. Gibbons, S.L., Ebbeck, V., \& Weiss, M.R. (1995). Fair play for kids: Effects on the moral development of children in physical education. Research quarterly for exercise and sport, 66(3), 247-255.

42. Giménez, J., Abad, M., \& Robles, J. (2009). La enseñanza de deporte desde la perspectiva educativa. Wanceulen E.F. digital, 5. Recuperado el 16 de febrero de 2018 de http://rabida.uhu.es/dspace/bitstream/handle/10272/3316/b15548818.pdf

43. Goldstein, J.D., \& Iso-Ahola, S.E. (2006). Promoting Sportmanship in Youth Sports. Journal of Physical Education, Recreation y Dance, 77(7), 18-24.

44. Gómez, A. (2007). La violencia en el deporte. Un análisis desde la Pedagogía Social. Revista de Pedagogía Social, 22(1), 63-87.

45. González, M.D. (2008). El deporte escolar en la Comunidad Autónoma de Madrid: Intervención didáctica y recursos humanos en las actividades físico-deportivas extraescolares en los centros educativos (Tesis Doctoral no publicada). Universidad de Valencia, España.

46. Guivernau, M., \& Duda, J. L. (2002). Moral atmosphere and athletic aggressive tendencies in young soccer players. Journal of Moral Education, 31(1), 67-85.

47. Hopkins, W.G. (2006). A new view of statistics: Effect magnitudes. Recuperado de: http://www.sportsci.org/resource/stats/effect. html\#effectsize

48. Kavussanu, M. (2006). Motivational predictors of prosocial and antisocial behaviour in football. Journal of Sport Sciences, 24, 575-588.

49. Lee, M. (1996). Young People, Sport and Ethics: An examination of fairplay in youth sport. London: Technical Report to the Research Unit of the Sports Council.

50. Lemyre, P.N., Roberts, G.C., \& Ommundsen, Y. (2002). Achievement goal orientations, perceived ability and sportspersonship in youth soccer. Journal of applied sport psichology, 14, 120-136.

51. Ligabrave. (2015). Área Psicoeducativa [Página web]. Recuperado de 25 de marzo de 2018 de http://ligabrave.es/area-psicoeduativa/

52. McAuley, E., Duncan, T.E., \& Tammen, V.V. (1989). Causal attributions and affective reactions to disconfirming outcomes in motor performance. Journal of Sport and Exercise Psychology, 11, 187-200.

53. Méndez-Giménez, A., Fernández-Río, J., \& Cecchini, J.A. (2016). El modelo de Vallerand en adolescentes asturianos: implementación y extensión. Revista Internacional de Medicina y Ciencias de la Actividad Física y del Deporte, 64, 703-722.

54. Miller, S.C., Bredemeier, B.J., \& Shields, D.L. (1997). Sociomoral education through physical education with at-risk children. Quest, 49, 114-129.

55. Nicholls, J.G. (1984). Achievement motivation: Conceptions of ability, subjective experience, Task choice, and performance. Psychological Review, 91, 328-346.
56. Nicholls, J.G. (1989). The competitive Ethos and Democratic Education. Cambridge, MA: Harverd University Press.

57. Ommundsen, Y., Roberts, G., Lemyre, P., \& Treasure, D. (2003). Perceived motivational climate in male youth soccer: relations to socio-moral functioning sportpersonship and team norm perceptions. Psychology of Sport and Exercise, 4(4), 397-413. doi:10.1016/S14690292(02)00038-9

58. Orts, F. (2013). El derecho educativo del menor en el deporte escolar (Tesis Doctoral no publicada). Universitat de Lleida, Espańa.

59. Pinheiro, V., Cameiro, O., \& Sequeira, P. (2014). El fair play en la iniciación deportiva, un estudio con entrenadores de fútbol. RETOS Nuevas Tendencias en Educación Física, Deporte y Recreación, 25, 32-35.

60. Pinheiro, V. (2013). O comportamento do treinador em competição na promoção do fair play. Um estudo nos escalóes de Futebol de Formação (Tesis doctoral no publicada), Universidad de Lleida, España.

61. Roberts, G.C., \& Balagué, G. (1991). The development and validation of the Perception of Success Questionnaire. Paper presented at the FEPSAC Congress, Cologne, Germany

62. Roberts, G.C., Treasure, D.C., \& Balagué, G. (1998). Achievement goals in sport: The development and validation of the Perception of Success Questionnaire. Journal of Sport Sciences, 16, 337 - 347.

63. Sáenz Ibáñez, A., Gimeno Marco, F., Gutiérrez Pablo, H., \& Garay Ibáñez de Elejalde, B. (2012). Prevención de la agresividad y la violencia en el deporte en edad escolar: Un estudio de revisión. Cuadernos de Psicología del Deporte, 12(2), 57-72.

64. Sampol, P.P., Rotger, P.A., Verdaguer, F.X., Conti, J.V., \& Álvarez, M.T. (2007). Intervención para la promoción de actitudes de fairplay en futbolistas cadetes. Apunts. Educación física y deportes, 3(89), 15-22.

65. Schwamberger, B.M., \& Curtner-Smith, M. (2016). Enhancing Sporting Behavior and Fair Play in Sport Education. Research Quarterly for Exercise and Sport, 87(S2), A103.

66. Smith, M. (1988). Interpersonal sources of violence in hockey: The influence of parents, coaches, and teammates. En F. L. Smoll, R.A. Magill, y M.J Ash. Children in sport (pp. 301-313). Champaign, IL. Human Kinetics.

67. Stephens, D.E. (2000). Predictors of likelihood to aggress in youth soccer: An examination of coed and all-girls teams. Journal of Sport Behavior, 23, 311-325.

68. Stornes, T., \& Ommundsen, Y. (2004). Achievement goals, motivational climate and sportspersonship: a study of young handball players. Scandinavian Journal of Educational Research, 48(2), 205-221

69. Treasure, D.C., \& Roberts, G.C. (1994). Perception of success questionnaire: Preliminary validation in an adolescent population. Perceptual and Motor Skills, 79, 607-610.

70. Vidoni, C., \& Ward, P. (2009). Effects of fair play instruction on student social skills during a middle school sport education unit. Physical Education and Sport Pedagogy, 14(3), 285-310.

71. Vilches, F. (2004). El menosprecio de la lengua. El español en la prensa. Madrid: Dykinson.

72. Weinberg, R., \& Gould, D. (2010). Fundamentos de Psicología del Deporte y el Ejercicio Físico. Madrid: Editorial Médica Panamericana.

73. White, S.A., \& Duda, J.L. (1994). The relationship of gender, level of sport involvement, and participation motivation to task and ego orientacion. International Journal of Sport Psychology, 25, 4-18. 